\title{
A SIMULATION STUDY OF PATH AND SPEED THROUGH DOUBLE-LANE ROUNDABOUTS
}

\author{
Gregory W. Davis \\ FHWA Office of Safety RD\&T \\ Human Centered Systems Team \\ McLean, VA 22101 \\ E-mail: gregory.davis@fhwa.dot.gov \\ Vaughan W. Inman, Ted Shafer, and Bryan J. Katz \\ SAIC Transportation Research Division \\ Human Centered Systems Team \\ McLean, VA 22101
}

\begin{abstract}
Summary: This study assessed the effects of geometric curvature and lane demarcation on drivers' selection of path and speed in double-lane roundabouts. By means of a PC-based simulator, path and speed data were collected as subjects drove twice through six roundabouts. The six roundabouts varied in terms of pavement markings and geometric curvature on the entry and exit. Seventy-five participants were tested using a fixed-base driving simulator. The results showed that drivers maintained lane position better when the roundabouts had lane demarcation than when the roundabouts had no lane demarcation. Furthermore, lane-tracking behavior for participants exposed to roundabouts with pavement markings was similar to lane-tracking behavior observed in a recent field study. Observations of speed indicated that drivers drove faster though roundabouts with a large central island radius as opposed to a roundabout with a smaller central island radius.
\end{abstract}

\section{INTRODUCTION}

Roundabouts are circular intersections with specific traffic control and geometric design features including: yield at entry, channelized approaches and exits, and appropriate geometric curvature to ensure slow speeds. Not to be confused with the older style traffic circles that allow entering traffic to have the right-of-way, modern roundabouts offer significant operational and safety improvements when compared to 4-way signalized intersections. Most modern U.S. roundabouts are designed to handle a single lane of traffic. To increase capacity, double-lane roundabouts can be used. However, the addition of a lane in the circle creates the potential for cars to cross paths (see Figure 1). Additionally, double-lane roundabouts may contain inadequate deflection on the approach, encouraging larger speed differentials between entering and circulating traffic.

In order to reduce speeds on entry, small radii curves may be used on the approach. These curves are tailored to slow entering vehicles to the design speed of the circulatory roadway. However, at double-lane roundabouts, small radii entry curves may induce vehicles in the left lane to swing to the right and "cut the corner" (Robinson et al., 2000). In doing so, the driver creates a straighter path of travel that allows for higher speeds and an increased risk to safety (Robinson et al., 2000). There are two common approaches for radii treatments on entry: a large-to-small radius 
and a small-to-large radius. A large-to-small radius begins with a gentle deflection to the right, gradually becoming sharper at the edge of the circulatory roadway. A small-to-large radius begins with a sharp deflection and then flattens to a gentle curve at the edge of the circulatory roadway.

In order to minimize path overlap, lane demarcation lines may be used on the approach and inside the circulatory roadway. The literature, however, seems divided concerning recommendations for roundabout lane demarcations inside the circulatory roadway. For example, Robinson et al. (2000) suggests that pavement marking in the circulatory roadway should not be used because it may provide the driver with a false sense of security. On the other hand, Arndt and Troutbeck (1998) assert that when pavement markings are present in the circulatory roadway, drivers have a lesser tendency to take overlapping paths because "they can see the edge of their lane." The current study was designed to assess the effects of geometric curvature and pavement markings on drivers' selection of path and speed through double-lane roundabouts. The study was performed on a desktop driving simulator.

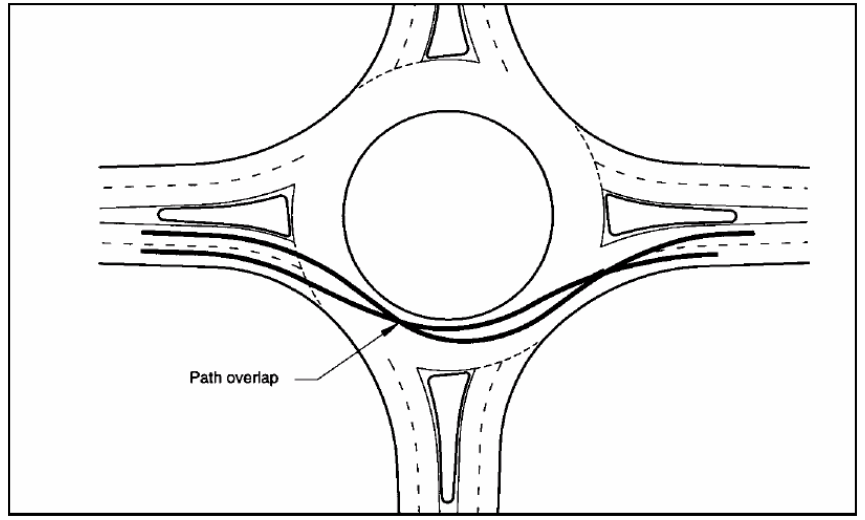

Figure 1. An example of path overlap in a double-lane roundabout

\section{METHOD}

\section{Simulator}

A fixed-based STISIM Model 500 driving simulator was used for the experiment. A customized driving buck, containing a force feedback steering wheel and pedals, was positioned in front of a 19" CRT monitor. The car seat was adjusted for each driver so that the eye point was 24 "' from the face of the monitor and level with the top of the monitor bezel. The roundabouts, signs, and roadway pavement markings were designed in 3-D Studio, and then imported as polygons into the STISIM Drive operating system. Sounds provided feedback for running off the road or hitting the center island of the roundabout.

\section{Participants}

Seventy-five male and female participants were recruited from the Washington, D.C. metropolitan area. All subjects were licensed drivers whose ages ranged from 19 to 81 . Demonstration of at least 20/40 vision using a Snellen eye chart was mandatory for proceeding with the experiment. Additionally, participants were screened for a history of motion sickness. 
Prior to experimentation, participants were given oral and written information concerning the general operational characteristics of a single-lane roundabout.

\section{Procedure}

Prior to data collection, all participants were given three training sessions. These sessions provided a hands-on learning experience for acceleration/braking, negotiating curves, and driving single-lane roundabouts, respectively. Participants were required to demonstrate a mastery of these skills in the simulator before proceeding to the double-lane roundabout test trials. These roundabouts included: (1) an existing urban roundabout with lane demarcations in the circulatory roadway, (2) the same urban roundabout without demarcations, (3) an existing interchange roundabout without lane demarcations, (4) the same interchange roundabout with lane demarcations in the circulatory roadway, (5) a customized double-lane roundabout with large-to-small curve entry radii, and (6) a customized double-lane roundabout with a small-tolarge entry radii. Participants drove twice through each of the roundabouts, once starting in the left lane, and again from the right lane. A Latin square was implemented for counterbalancing the trials. The dependent measures of lane position and speed were measured at seven locations in the roundabout (Figure 2). Lane position was scored using a 9-point scale (Table 1).

Independent within-subjects variables included: Roundabout Type (Urban/Interchange), Entry Radii (large-small or small-large), Start Lane (left/right), and Measurement Location (1-7). Only Roundabouts 5 and 6, adapted from Robinson et al. (2000), were evaluated for Entry Radii.

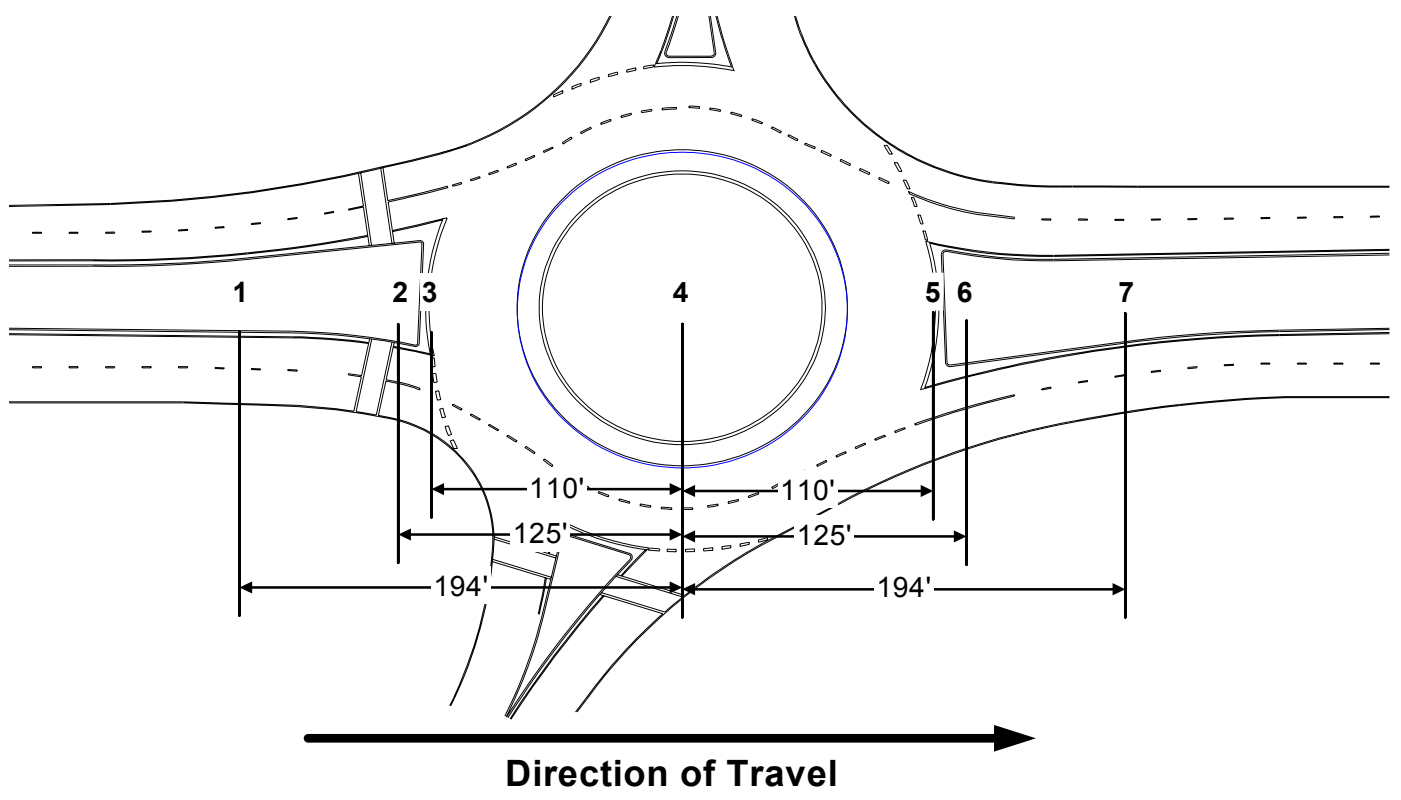

Figure 2. Speed and Lane Position were measured at 7 locations in the simulated roundabouts. 
Table 1. Lane Position scoring criterion

\begin{tabular}{|c|l|c|l|}
\hline Score & Lane Position & Score & Lane Position \\
\hline 0 & Out of left-lane to left & 5 & Right-lane encroaching on left-lane \\
1 & Encroaching on left-lane edge & 6 & Centered in right-lane \\
2 & Centered in left-lane & 7 & Encroaching on right-lane edge \\
3 & Left-lane encroaching on right-lane & 8 & Out of lane to right \\
4 & Straddling lanes & & \\
\hline
\end{tabular}

\section{RESULTS}

\section{Analysis of Speed}

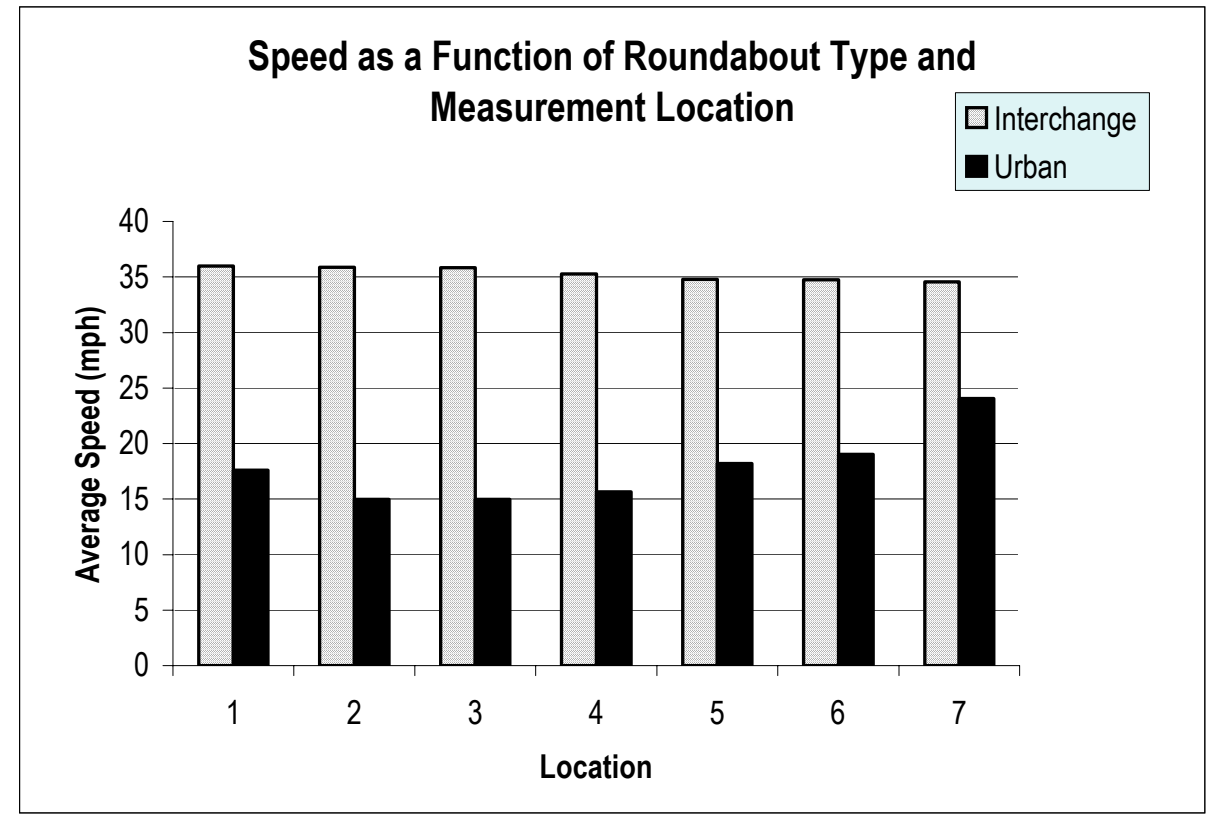

Figure 3. Participants drove faster through the larger Interchange Roundabout (35.3 mph) than the smaller Urban Roundabout (17.8 mph). 
Analysis of Path

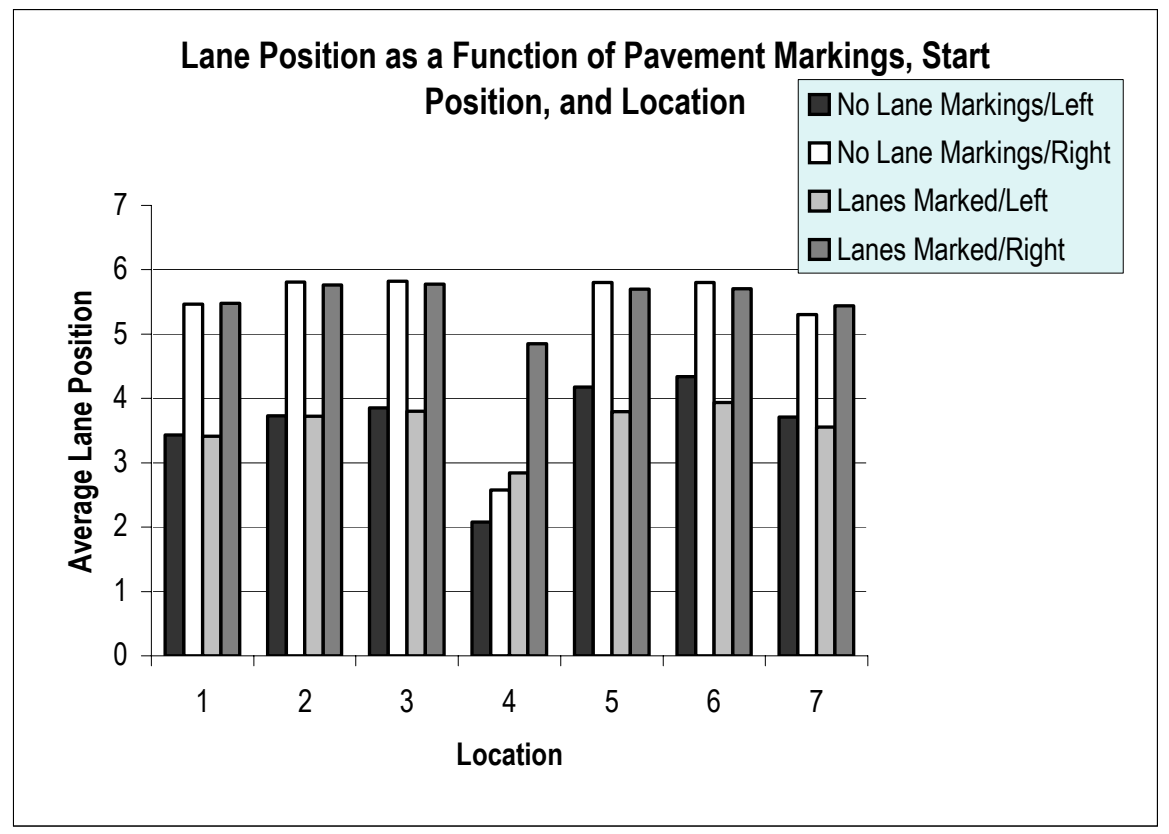

Figure 4. For drivers starting in the right lane, the presence of pavement markings prevented them from drifting to the left lane inside the circulatory roadway at Location 4 - a potential safety benefit in the field.

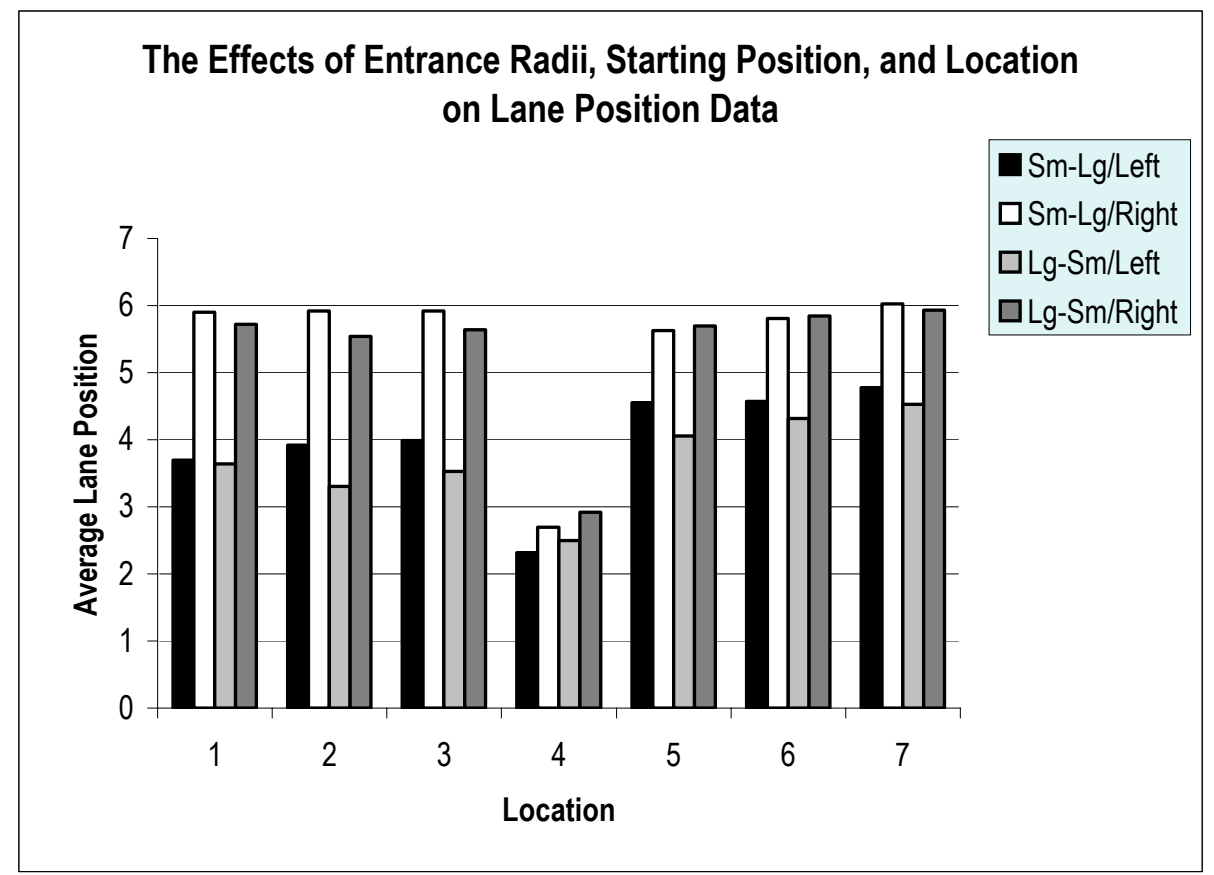

Figure 5. Entry radii had a small effect on lane positions at the $2^{\text {nd }}$ and $3^{\text {rd }}$ locations, with the large-small condition displacing subjects slightly to the left on the roadway. 


\section{DISCUSSION}

Measurements of driving on speed for Roundabouts 1-4 indicated that participants, regardless of starting lane position or the presence of pavement markings, drove faster through the Interchange Roundabouts. Note in Figure 3 that the speeds for the Interchange Roundabout remain relatively constant, whereas drivers in the Urban Roundabout reduce their speeds in the center of the roundabout, and then accelerate on the exit. The larger size of the Interchange Roundabout relative to the Urban Roundabout explains the higher observed speeds, allowing drivers more room to negotiate curves with larger radii. An analysis of path data for the same roundabouts (Figure 4) indicates that the absence of pavement markings had a profound effect on lane position data, at Location 4, when participants started in the right lane. Without pavement markings, subjects crossed over to the left lane while traversing the circulatory roadway. The large-to-small entry shifted the lane position of all drivers, regardless of starting lane, slightly to the left compared to the small-to-large entry. Because drivers in both lanes shifted about the same amount, it is doubtful that this effect, if validated in the field, would have an effect on safety. The effects of Entry Radii on Speed were not significant. This study suggests that pavement markings may improve safety by reducing potential vehicle-vehicle conflicts.

\section{REFERENCES}

Robinson, B. W., Troutbeck, R., Werner, B., Lothar, B., Courage, K., Kyte, M., Mason, J., Flannery, A., Myers, E., Bunker, J., Jacquemart, G. (2000). Roundabouts: an Informational Guide. FHWA-RD-00-067. Federal Highway Administration, McLean, VA.

Arndt, O.K. and Troutbeck, R. J. (1998). Relationship between roundabout geometry and accident rates. Transportation Research Circular E-C0002. Washington, D.C.: TRB. 